\title{
Article \\ Propositions for Confidence Interval in Systematic Sampling on Real Line
}

\author{
Mehmet Niyazi Çankaya \\ Department of Statistics, Faculty of Arts and Science, University of Uşak, Ankara-İzmir Karayolu 8.Km. \\ 1.Eylül Kampüsü UŞAK - 64200, Turkey; mehmet.cankaya@usak.edu.tr; Tel.:+ 092762212121 - 2585
}

\begin{abstract}
The systematic sampling is used as a method to get the quantitative results from the tissues and the radiological images. Systematic sampling on real line $(\mathbb{R})$ is a very attractive method within which the biomedical imaging is consulted by the practitioners. For the systematic sampling on $\mathbb{R}$, the measurement function (MF) is occurred by slicing the three-dimensional object equidistant systematically. The currently used covariogram model in variance approximation proposed by $[28,29]$ is tested for the different measurement functions in a class to see the performance on the variance estimation of systematically sampled $\mathbb{R}$. This study is an extension of [17], and an exact calculation method is proposed to calculate the constant $\lambda(q, N)$ of confidence interval in the systematic sampling. The exact value of constant $\lambda(q, N)$ is examined for the different measurement functions as well. As a result, it is observed from the simulation that the proposed $M F$ should be used to check the performances of the variance approximation and the constant $\lambda(q, N)$. Synthetic data can support the results of real data.
\end{abstract}

Keywords: biomedical imaging; covariogram; design-based stereology; estimation of volume; systematic sampling

\section{Introduction}

The systematic sampling, often used in the area of biomedical imaging, is a design-based approach for estimating a parameter $Q$ of the geometrical quantities, such as volume, area, surface area, length. In the systematic sampling principle, geometrical objects are sampled with probes, such as lines, regular grid, or designed patterns. The probes superimposed on the geometrical objects are tools for us to get the quantitative values of geometrical objects. If we have an equidistant systematically sampling on geometrical objects, we will have the estimated values for each replication of sampling of these objects. The estimated values obtained from each replication of this sampling on geometrical objects produce a fluctuation $[14,17,18,24,32]$. So, this fluctuation is modelled by Fourier transformation $\mathcal{F}_{1}$, which was considered by $[28,29]$. Since we have the estimated values for parameter $Q$, the variance estimation is needed. For the systematic sampling on $\mathbb{R}$, also called as Cavalieri sampling, previous studies shed some light on the variance estimation for the systematic sampling on real line, $[9,16,19,20,25-27,30]$. Main source in these studies is of inspiration in Matheron's theory. Matheron proposed his covariogram model $g(h)$ intuitively. In this study, we aim to focus on testing the performance of this covariogram model for the different measurement functions in the regular and irregular forms. It is important to test the capability of fitting performance of his covariogram model, because the sampling directions (axises) which are axial, coronal or sagittal produce the different measurement functions, as implied by [17]. There is no study addressing the performance evaluation of the covariogram model for the different measurement functions. In this context, the papers $[2-5,14,15,18,20,21,23,24,31,36-39]$ used the Matheron's covariogram model for the different measurement functions. 
After having the estimated value for the parameter $Q$, said as $\hat{Q}$, we should focus on the confidence interval for $\hat{Q}$. [17] proposes two approaches for the coefficient of confidence interval. The first one is based on the statistical theory. In this study, the second proposition of [17] is examined. Main motivation is to find a calculation method for the constant $\lambda(q, N)$ which is used to construct confidence interval for $\hat{Q}$. In this sense, we will give the exact calculation method for $\lambda(q, N)$, so we have more accurate information for the confidence interval. We also test the exact values of $\lambda(q, N)$ for the different measurement functions.

The paper is organized as follows. Section 2 introduces the materials in the systematic sampling on $\mathbb{R}$ and describes the exact calculation method for constant $\lambda(q, N)$ as well. A simulation study and real data examples are given at Section 3. A section 4 is considered for the discussions on results.

\section{Materials and Methods}

The estimation for volume $Q$, the empirical true variance, the true variance, the variance approximation, and the confidence interval for systematic sampling on $\mathbb{R}$ with variance approximation formula are introduced briefly.

\subsection{Estimations for Volume $Q$ and Variance of $\hat{Q}$}

\subsubsection{Estimation for Volume $Q$}

Suppose that we have three-dimensional geometrical object. This object is a fixed, bounded, nonvoid with piecewise smooth boundary of finite surface area of $Q$, except fractals. The volume value of the object is parameter $Q$. Let parameter $Q$ be estimated. To get the estimation value for the parameter $Q$, Cavalieri planes called as the measurement function $f$ are used. The function $f$ has to represent the shape of the geometrical object. The mathematical expression for the volume estimation with Cavalieri planes is given in the following form,

$$
\hat{Q}(\mathbf{u}, T)=T \sum_{j=0}^{n-1} f((\mathbf{u}+j) T) .
$$

$T$ is a constant distance among the slices obtained from three-dimensional object. $\mathbf{u}$ is an uniform random variable in the interval $[0, T)$. $\hat{Q}$ depends on the random variable $\mathbf{u}$ and the slice thickness or the number of systematic sampling, $n$.

The problem is about predicting $\operatorname{Var}(\hat{Q})=\mathbb{E}(\hat{Q}-Q)^{2}=\mathbb{E}\left(\hat{Q}^{2}\right)-Q^{2}$. Since the uniform distribution defined at $[0, T)$ is used, $\mathbb{E}\left(\hat{Q}^{2}\right)=1 / T \int_{0}^{T} \hat{Q}^{2} d u$. In following subsections, we will introduce some important formulae for the variance estimation and its counterparts $[6,9,12,16]$.

\subsubsection{Variance of $\hat{Q}$}

The behavior of the variance of the Cavalieri estimator is strongly connected to analytical properties of the measurement function $f$. An aspect coming from Matheron's transitive theory is given in [9] for these properties. The variance of Cavalieri sampling changes with a fractional power of $T[16,17]$. The fact that there is a fractional power for $T$ is not pointed out by $[26,27]$. In this sense, [16] is an extension of [26]. For this reason, we want to test the performance of covariogram model in Eq. (11) and the variance extension term in Eq. (19) for the different measurement functions.

For the practical purpose, since the true $M F$ is not known, we do not get the true variance of systematic sampling on $\mathbb{R}$. For this reason, the variance approximation known as a variance extension $\left(\operatorname{Var}_{E}(\hat{Q})\right)$ term is given. It is in the two forms coming from Fourier transformation basically given in [9] and the properties of measurement functions proposed by [16].

Firstly, we will introduce the empirical true variance for $\hat{Q}$. Secondly, the true variance for $\hat{Q}$ is defined in Eq. (4). It includes the covariogram function. After the covariogram function is defined, the measurement functions will be introduced. 

79 below,

The empirical true variance of systematic sampling on $\mathbb{R}$ is calculated by the formula given

$$
\operatorname{Var}(\hat{Q})=\frac{1}{m} \sum_{r=1}^{m}\left(\hat{Q}_{r}-Q\right)^{2},
$$

where

$$
g(h)=\int_{\mathbb{R}} f(x) \cdot f(x+h) d x, h \in \mathbb{R}
$$

${ }_{91}$ is a covariogram function of the measurement function $f$. It is proposed by Matheron's transitive

$$
G(t)=\mathcal{F}_{1} g(h)=\int_{-\infty}^{\infty} g(h) \exp (-2 \pi i t h) d h .
$$

94 Note that subscript is for the dimension. In this case, we are interested in the one dimensional 95 systematic sampling.

Suppose that we have measurement functions in Eqs. (7)-(10). These functions are used for the simulation. They can represent the biological objects. They are given with following forms and the function $f$ has positive values for each values of variable $x$, namely $f: \mathbb{R} \rightarrow \mathbb{R}^{+}$.

$$
\begin{gathered}
f(x)=\left(1-x^{2}\right)^{q}, x \in[-1,1], q \in[0,1], \\
f(x)=\left((1-\cos (x))\left(1-x^{2}\right)\right)^{q}, x \in[-1,1], q \in[0,1],
\end{gathered}
$$

where $q$ is a parameter of smoothness constant.

$$
f(x)=\exp \left(-\sin \left(-x^{3}\right)\right), x \in[-38 \pi / 100,53 \pi / 100],
$$

and

$$
f(x)=(5 / 112)\left(-54 x^{4}-25 x^{3}+48 x^{2}+25 x+6\right), x \in[-1,1] .
$$

96 They represent the area measured on the each slices of three-dimensional biological objects. The measurement functions in Eqs. (7) and (10) are used by [16,22], respectively. Each function $f$ and its 
covariogram function can be an example for the representation of $q$ even though the function $f$ does not have the parameter $q$. Two approximately same functions can be proposed, $f_{i} \approx f_{j}$, where $i \neq j$, $i, j=\mathbb{N}$, which have the different mathematical expressions, however they can be a neighborhood to each other. For example, the measurement functions in Eqs. (9) - (10) can be more modified version of $M F$ in Eq. (8). $M F$ in Eq. (8) can be a modified version of $M F$ in Eq. (7).

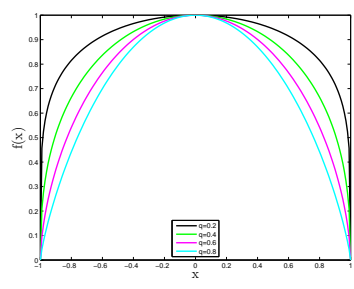

(a) MF of Eq. (7)

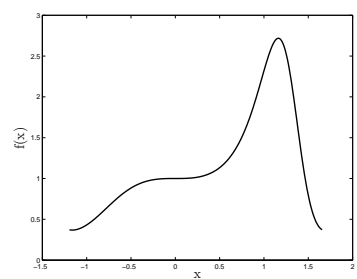

(a) MF of Eq. (9)

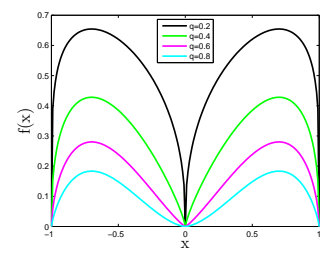

(b) MF of Eq. (8)

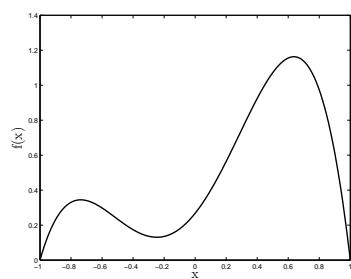

(b) MF of Eq. (10)

Since the covariogram functions of measurement functions in Eqs. (7)-(10) can not be calculable to get the integral values, a model for the covariogram functions should be proposed. In this sense, the covariogram functions can be modelled by a polynomial with the fractional power,

$$
g(h)=b_{0}+b_{j}|h|^{j}+b_{2} h^{2}, j=2 q+1, q \in[0,1] .
$$

111

Eq. (11) is defined to be a covariogram model $[6,9,16,28,29]$.

\subsubsection{Approximate Variance of $\hat{Q}$}

By using Eq. (4) and the properties of $G(t)$, we finally get

$$
\operatorname{Var}(\hat{Q})=T^{-1} \int_{0}^{T} \hat{Q}^{2} d u-Q^{2}=\sum_{k=-\infty}^{\infty} G(k / T)-G(0)=2 \sum_{k=1}^{\infty} G(k / T)
$$

(Detailed expressions are given in [9,16,28,29]).

The Fourier transformations of parts $h^{0}$ and $h^{2}$ in the covariogram model in Eq. (11) are zero. Thus, the variance extension term of systematic sampling on $\mathbb{R}$ is obtained by using the formula given in Eq. (9.1) and Eq. (9.2) in [9] as follow,

$$
\mathcal{F}_{1}|h|^{j}=b(j, 1) \varrho^{-(j+1)},(j>-1, \text { non-even })
$$

which is the Fourier transformation of $|h|^{j}$ for the one dimensional systematic sampling. $b(j, 1)=$ $\pi^{-j-1 / 2} \frac{\Gamma\left(\frac{j+1}{2}\right)}{\Gamma\left(-\frac{j}{2}\right)}$. 
By using Eq. (11), Eq. (12), and Eq. (13), Eq. (14) is obtained.

$$
\begin{aligned}
\operatorname{Var}_{E}(\hat{Q}) & =2 b_{j} b(j, 1) T^{j+1} \zeta(j+1) \\
\operatorname{Var}_{E}(\hat{Q}) & =\alpha(q)(3 g(0)-4 g(T)+g(2 T)) \\
\operatorname{Var}_{E}(\hat{Q}) & =\alpha(q)\left(3 C_{0}-4 C_{1}+C_{2}\right) T^{2}
\end{aligned}
$$

where

$$
\begin{gathered}
\alpha(q)=\frac{2 \pi^{-(2 q+3 / 2)} \Gamma(q+1) \zeta(2 q+2)}{\left(2^{2 q+1}-4\right) \Gamma\left(-\frac{2 q+1}{2}\right)}, q \in \mathbb{R}, \\
\hat{g}(k T)=T C_{k},
\end{gathered}
$$

$\Gamma, \zeta$ are Gamma and Riemann's Zeta functions in [1], respectively. $\hat{g}(k T)$ is an unbiased point estimators of $g(k T), k=0,1,2, \ldots$.

Eq. (15) is gotten according to Fourier transformation basically given in [9]. However, Eq. (17) is obtained according to the generalized version of refined Euler-MacLaurin, proposed by [16].

$$
\begin{gathered}
\alpha(q)=\frac{\Gamma(2 q+2) \zeta(2 q+2) \cos (q \pi)}{(2 \pi)^{2 q+2}\left(1-2^{2 q-1}\right)}, q \in[0,1], \\
\operatorname{Var}_{E}(\hat{Q})=\frac{2 \pi^{-(2 q+3 / 2)} \Gamma(q+1) \zeta(2 q+2)}{\left(2^{2 q+1}-4\right) \Gamma\left(-\frac{2 q+1}{2}\right)}\left(3 C_{0}-4 C_{1}+C_{2}\right) T^{2} .
\end{gathered}
$$

Note that Eq. (15) and Eq. (17) give the same results, because the connection between the generalized version of refined Euler-MacLaurin summation formula and the Matheron's transitive theory is expressed by [16].

We calculate the estimated coefficient of error for the systematic sampling on $\mathbb{R}$ by means of Eq. (19) given below,

$$
\begin{aligned}
{\widehat{v a r_{E}}}_{(\hat{Q}) / Q^{2}} & =\alpha(q)\left(3 C_{0}-4 C_{1}+C_{2}\right) T^{2} / Q^{2} \\
\hat{c} e(\hat{Q}) & =\sqrt{\alpha(q)\left(3 C_{0}-4 C_{1}+C_{2}\right)}\left(\sum_{i=1}^{n} f_{i}\right)^{-1}
\end{aligned}
$$

where

$$
C_{k}=\sum_{i=1}^{n-k} f_{i} \cdot f_{i+k}, k=0,1, \ldots, n-1 .
$$

This is defined to be a coefficient of error of Matheron's covariogram model. Eq. (5) and Eq. (20) have in fact inheritance. $n \geq 2 k+1$ observations are required due to Eq. (20) $[9-11,15,16,28,29]$.

The estimation values of parameter $q$ from $\left(1-x^{2}\right)^{q},\left((1-\cos (x))\left(1-x^{2}\right)\right)^{q}, \exp \left(-\sin \left(-x^{3}\right)\right)$ and $(5 / 112)\left(-54 x^{4}-25 x^{3}+48 x^{2}+25 x+6\right)$ measurement functions will be obtained at the simulation section. $[2-5,14,15,18,20,21,23,24,31,36-39]$ used currently the covariogram model for the different measurement functions. We aim to show the performance of the covariogram model in Eq. (11) for the different measurement functions. In other words, the capability of covariogram model for fitting performance on the covariogram functions is tested, so the information gained by this model is displayed by the simulation results. By using this model and the variance extension term in Eq. (14), the estimation formula of parameter $q$ is given into following subsection.

\subsubsection{Estimation Formula of Parameter $q$}

The estimation formula of smoothness constant $q$ is proposed by [16]. In this subsection, we will get it via the variance extension term in Eq. (14) in the framework of Fourier transformation. The formula we got for $q$ is the same with the formula in [16]. 
The covariogram model $g$ can be declared by integer $k$ values. If $h=i T$ is near zero, Eq. (6) has more information. By using Eqs. (11) and (14), respectively,

$$
g(i T)=b_{0}+b_{2 q+1}(|i T|)^{2 q+1}+b_{2}(i T)^{2}, i=0, k, 2 k,
$$

$$
\begin{aligned}
\operatorname{Var}_{E}(\hat{Q})= & 2 b_{2 q+1} b(2 q+1,1) T^{2 q+2} \zeta(2 q+2) \\
\operatorname{Var}_{E}(\hat{Q})= & 2 \pi^{-(2 q+3 / 2)} \frac{\Gamma(q+1)}{\Gamma\left(-\frac{2 q+1}{2}\right)} \zeta(2 q+2) \\
& \frac{T \cdot 3 g(0)-4 g(k T)+g(2 k T)}{k^{2 q+1}\left(2^{2 q+1}-4\right)}
\end{aligned}
$$

can be obtained. By using Eq. (18) and Eq. (21),

$$
\frac{1}{k^{2 q+1}}[3 g(0)-4 g(k T)+g(2 k T)]=[3 g(0)-4 g(T)+g(2 T)]
$$

is obtained and then

$$
q=\frac{1}{2 \log (k)} \cdot \log \left(\frac{3 g(0)-4 g(k T)+g(2 k T)}{3 g(0)-4 g(T)+g(2 T)}\right)-\frac{1}{2}
$$

where, $k=2,3, \ldots$.

can be obtained. By using Eq. (16),

$$
\hat{q}=\frac{1}{2 \log (k)} \cdot \log \left(\frac{3 C_{0}-4 C_{k}+C_{2 k}}{3 C_{0}-4 C_{1}+C_{2}}\right)-\frac{1}{2}, k=2,3, \ldots .
$$

The estimator $\hat{q}$ is gotten when the $M F$ is obtained by planimetry. The planimetry is also called as an automatic pixel counting given in the section for real data.

\subsection{Confidence Interval in Systematic Sampling on $\mathbb{R}$}

We will give an exact calculation of $\lambda(q, N)$ in a generalized version of the refined Euler-MacLaurin summation formula with a fractional power of measurement functions. A brief introduction, and some tools will be given in next subsection to get the formula of $\lambda(q, N)$ for the confidence interval of $\hat{Q}$.

\subsubsection{Confidence Interval of $\hat{Q}: \lambda(q, N)$}

Some definitions expressed in [16] and [17] will be given. A theorem will be proposed for a tool in the constant $\lambda(q, N)$.

A bounded interval for the difference $(\hat{Q}-Q)$, which is defined as a generalized version of the refined Euler-MacLaurin summation formula, is

$$
|\hat{Q}-Q| \leq T^{q+1} P_{q+1}^{*} \sum_{i=1}^{N}\left|S f^{(q)}\left(a_{i}\right)\right|
$$

where

$$
P_{q+1}^{*}=\max _{\{\Delta, \beta\}}\left\{\left|\frac{-2}{(2 \pi)^{q+1}} \sum_{j=1}^{\infty} \frac{1}{j^{q+1}} \cos \left(2 \pi \Delta j-\frac{\pi}{2}(q+1)+\beta\right)\right|\right\},
$$

$\Delta \in[0,1)$ and $\beta \in[0,2 \pi] .\left\{a_{i}, i=1,2, \ldots, N\right\}$ is the set of real line at which function $f^{(q)}$ exhibits discontinuities. $N$ is the number of set. The difference $\hat{Q}-Q$ and $\left(S f^{(q)}\left(a_{i}\right)\right)^{2}$ are given by Eq. (6) and Eq. (11) in [17]. If $N$ is greater than 2, the information in $\hat{Q}-Q$ decreases (For detailed 
We need the following periodic function with period $T$ to get the values of constant $\lambda(q, N)$

where

$$
\begin{aligned}
\frac{|\hat{Q}-Q|}{T^{q+1} P_{q+1}^{*}} & \leq \sum_{i=1}^{N}\left|S f^{(q)}\left(a_{i}\right)\right| \\
|\hat{Q}-Q| & \leq T^{q+1} P_{q+1}^{*} \sqrt{N} \sqrt{\sum_{i=1}^{N}\left(S f^{(q)}\left(a_{i}\right)\right)^{2}}
\end{aligned}
$$

is obtained.

The following formula having the parameter $q$ is proposed by [16],

$$
\operatorname{Var}_{E}(\hat{Q})=T^{2 q+2} \frac{P_{2 q+2, T}(0)}{\cos (\pi q)} \sum_{i=1}^{N}\left(S f^{(q)}\left(a_{i}\right)\right)^{2}
$$

When $\sum_{i=1}^{N}\left(S f^{(q)}\left(a_{i}\right)\right)^{2}$ in Eq. (27) is written in Eq. (26), Eq. (28) is obtained.

$$
\begin{gathered}
|\hat{Q}-Q| \leq P_{q+1}^{*} \sqrt{N} \sqrt{\frac{\cos (\pi q)}{P_{2 q+2, T}(0)}} \sqrt{\operatorname{Var}_{E}(\hat{Q})}, \\
|\hat{Q}-Q| \leq \lambda(q, N) \sqrt{\operatorname{Var}_{E}(\hat{Q})},
\end{gathered}
$$

$$
\lambda(q, N)=P_{q+1}^{*} \sqrt{N} \sqrt{\frac{\cos (\pi q)}{P_{2 q+2, T}(0)}}, q \in[0,1] .
$$

$\lambda(q, N)$ is a function of $q$ and $N$. In the following steps, we will give a definition for the function $P_{k, T}$ in [1]. The function $P_{q+1}^{*}$ in Eq. (24) is required to apply Theorem 2.1 for the exact calculation of $\lambda(q, N)$.

From Eq. (28), for true parameter $Q$ a bounded interval (or 100\% confidence interval) is given as $[17,18]$

$$
\left(\hat{Q}-\lambda(q, N) \sqrt{\operatorname{Var}_{E}(\hat{Q})}, \hat{Q}+\lambda(q, N) \sqrt{\operatorname{Var}_{E}(\hat{Q})}\right) .
$$
$[1,16]$,

$$
P_{k, T}(x)=P_{k}\left(\frac{x}{T}-\left[\frac{x}{T}\right]\right)=\frac{-2}{(2 \pi)^{k}} \sum_{j=1}^{\infty} \frac{\cos (2 \pi j(x / T)-(1 / 2) \pi k)}{j^{k}},
$$

\footnotetext{
where $x \in \mathbb{R}, k=2,3, \ldots$
}

expressions, see [17]). The preliminaries were given. Now, we will use the tools in [16,17]. After some By means of Cauchy Schwarz inequality $\left(\sum_{i=1}^{N}\left|y_{i}\right|\right)^{2} \leq N \sum_{i=1}^{N} y_{i}^{2}$, where $y_{i}=S f^{(q)}\left(a_{i}\right)$

When Eq. (23) and Eq. (25) are used

$$
\left(\sum_{i=1}^{N}\left|S f^{(q)}\left(a_{i}\right)\right|\right)^{2} \leq N \sum_{i=1}^{N}\left(S f^{(q)}\left(a_{i}\right)\right)^{2} .
$$


By using Eq. (34), for $q=0.1$,

$$
\lambda(0.1,2)=\lim _{h \rightarrow 0}\left|\frac{-2}{(2 \pi)^{1.1}} \sum_{j=1}^{\infty} \frac{1}{j^{1.1}} \cos \left(2 \pi(1-h) j-\frac{\pi}{2}(1.1)+2 \pi\right)\right| \cdot \sqrt{\frac{(2 \pi)^{2.2}}{\zeta(2.2)}}
$$$$
=2.71243
$$

3. Supposing that we have a left-open side and right-open side interval $(a, b)$, the maximum value of the interval is

$$
\begin{aligned}
& \lim _{h \rightarrow 0}(a+h)=\lim _{h \rightarrow \infty}(a+1 / h), \\
& \lim _{h \rightarrow 0}(b-h)=\lim _{h \rightarrow \infty}(b-1 / h) .
\end{aligned}
$$

In order to calculate $P_{q+1}^{*}$ in Eq. (24) exactly, $\Delta$ is replaced with $1-h$, because we must take the maximum value of $\Delta$. When $h \rightarrow 0$, we get the result for the function $P_{q+1}^{*}$ given in the following form,

$$
P_{q+1}^{*}=\lim _{h \rightarrow 0}\left|\frac{-2}{(2 \pi)^{q+1}} \sum_{j=1}^{\infty}\left[\frac{1}{j^{q+1}} \cos \left(2 \pi(1-h) j-\frac{\pi}{2}(q+1)+2 \pi\right)\right]\right| .
$$

Now we can explain how to get the values of constant $\lambda(q, N)$ below. In following lines, we will give the limit values done by Mathematica 7,8 or 9 .

When $N$ and $P_{2 q+2, T}(0)$ given in Eq. (29) are replaced with 2 and Eq. (32), respectively, we get an equation,

$$
\lambda(q, N)=P_{q+1}^{*} \sqrt{2} \sqrt{\frac{(2 \pi)^{2 q+2}}{2 \zeta(2 q+2)}} .
$$

The codes written in Mathematica 7 or higher versions 8 and 9 do not compute the value of $\lambda(0,2)$ and give infinity. So, for the calculation of $q=0$, we use Eq. (35) in order to give the result.

By means of Eq. (34), we get $\lambda(0,2)$ :

$$
\begin{aligned}
P_{q+1}^{*} & =\lim _{h \rightarrow 0}\left|\frac{-2}{(2 \pi)} \sum_{j=1}^{\infty} \frac{1}{j} \cos \left(2 \pi(1-h) j+\frac{3 \pi}{2}\right)\right|=1 / 2 \\
\lambda(0,2) & =\frac{1}{2} \cdot \sqrt{\frac{(2 \pi)^{2}}{\zeta(2)}}=2.44949 .
\end{aligned}
$$


is gotten. For other $q$ values, similar procedure is proceeded. By using the formula given in Eq. (29), the values at Table 1 are obtained for $N=2, N=3$ and $N=4$ [13].

It is attested that $\lim _{h \rightarrow \infty}(1-1 / h)$ and $\lim _{h \rightarrow 0}(1-h)$ give the same values for $\lambda(q, N)$, which is used to define the right-open side interval.

Moreover, in order to construct the confidence interval for the data set,

$$
\hat{Q}-\lambda(q, N) \cdot \hat{c} e(\hat{Q}) \cdot \hat{Q} \leq Q \leq \hat{Q}+\lambda(q, N) \cdot \hat{c} e(\hat{Q}) \cdot \hat{Q}
$$

are used, where $\lambda(q, N)$ is the coefficient of confidence interval. $\hat{c e}$ is an estimated coefficient of error $[17,18]$.

\subsubsection{Exact and Computational Values of $\lambda(q, N)$}

We got the values of $\lambda(q, N)$ for different $q$ and $N$ values by using the exact calculation. This calculation was done by the Mathematica 7 or higher versions 8 and 9 . One can get the values of $\lambda(q, N)$ for the different $q$ values with $N=2, N=3$, and $N=4$. When the number of sequence in codes prepared by [17] is increased, the computational values of $\lambda(q, N)$ convergence the exact values of $\lambda(q, N)$ (see Tables 1 and 2). Because getting the computational values of $\lambda(q, N)$ for $N=2$ would be unlikely to be useful, we give up computing some of them. $S_{i}$ : The number of sequence increased by a user, $i: 1,2,3,4$.

Table 1. $q$ and its exact $\lambda(q, N)$ values for different $N$ values

\begin{tabular}{|c|l|c|c|}
\hline$q$ & $\lambda(q, 2)$ & $\lambda(q, 3)$ & $\lambda(q, 4)$ \\
\hline \hline 0 & 2.44949 & - & - \\
\hline 0.1 & 2.71243 & 3.32203 & 3.83595 \\
\hline 0.2 & 2.93821 & 3.59855 & 4.15525 \\
\hline 0.3 & 3.12464 & 3.82689 & 4.41891 \\
\hline 0.4 & 3.26925 & 4.004 & 4.62342 \\
\hline 0.5 & 3.36968 & 4.12699 & 4.76544 \\
\hline 0.6 & 3.42394 & 4.19345 & 4.84218 \\
\hline 0.7 & 3.43064 & 4.20165 & 4.85165 \\
\hline 0.8 & 3.38906 & 4.15073 & 4.79285 \\
\hline 0.9 & 3.29929 & 4.04079 & 4.6659 \\
\hline 1 & 3.16228 & 3.87298 & 4.47214 \\
\hline
\end{tabular}

Table 2. $q$ and its computational $\lambda(q, N)$ values for $N=2$

\begin{tabular}{|c|c|c|c|}
\hline$S_{1}: \lambda(q, N)$ & $S_{2}: \lambda(q, N)$ & $S_{3}: \lambda(q, N)$ & $S_{4}: \lambda(q, N)$ \\
\hline \hline 2.402871 & 2.418465 & - & - \\
\hline 2.671264 & - & - & - \\
\hline 2.89612 & - & - & 2.926749 \\
\hline 3.07818 & 3.105091 & 3.111937 & 3.114245 \\
\hline 3.216635 & - & - & 3.258353 \\
\hline 3.323898 & - & - & 3.365038 \\
\hline 3.397839 & 3.408876 & 3.412577 & 3.414001 \\
\hline 3.418973 & - & - & 3.426855 \\
\hline 3.383912 & - & 3.38736 & 3.387638 \\
\hline 3.297052 & 3.29831 & 3.298648 & 3.298765 \\
\hline 3.161317 & - & - & - \\
\hline
\end{tabular}

\section{Results}

This section will give the simulation results for the estimation of parameter $q$ in the variance extension term in Eq. (19). Together with the estimated $\hat{q}$ with $k=2$ for Eqs. (7)-(10), it is planned to see whether or not the confidence interval includes the true value of volume. While constructing the confidence interval, the approximate variance based on the Matheron's covariogram model and the empirical true variance estimations are used. Real data examples are given to test the performance of confidence interval.

\subsection{Simulation}

\subsubsection{Plan and Output of Simulation}

CEMC, CEET, CPCEMC and CPCEET are the abbreviations for the coefficient of error of Matheron's covariogram model, the coefficient of error for the empirical true variance, the coverage probability of confidence interval based on the coefficient of error for the Matheron's covariogram model and the coverage probability of confidence interval based on the coefficient of error for the 
empirical true variance, respectively. The last two abbreviations are for the coverage probabilities of confidence intervals based on $C E M C$ and CEET. If the true value $Q$ is in the confidence interval, the confidence interval is successful. The numbers of success for each replication of sampling are counted and divided by the number of replication. Thus, the values of coverage probability of confidence interval for a parameter $Q$ are computed. $\widehat{\operatorname{Var}}(\hat{q})$ and $\widehat{M S E}(\hat{q})$ are the simulated variance and the simulated mean squared error of the estimator $\hat{q}$, respectively. The $k$ in $q$ formula was taken to be 2, because $h=i T$ must be near zero in order to increase the information in Eq. (6).

In the simulation performed, the number of replication is 3000 and the numbers of systematic sampling, $n$, on $\mathbb{R}$ are from 5 to 20 for the measurement functions in Eqs. (7)-(10). The number of systematic sampling is taken to be 20 at most for the practical purpose.

The following lines represent the producing $x$ values of $M F$, the values of $M F$, the estimated values of parameter $Q$ and the coefficient of error for the empirical true variance in three blocks given below.

\section{FOR $i$ FROM 1 TO the number of replication}

FOR $j$ FROM 0 TO the number of systematic sampling minus one

$T(j)=$ Length of interval of domain of function / the number of systematic sampling

FOR $k$ FROM 1 TO the number of systematic sampling

$x(k, j, i)=$ Left end point of the domain of the measurement function $+(u(j, i)+j) T(j)$

$y(k, j, i)=$ Measurement function $(x(k, j, i))$

\section{END FOR}

END FOR

END FOR

FOR $i$ FROM 1 TO the number of replication

FOR $j$ FROM 1 TO the number of systematic sampling

Estimated $Q$ in replicated sampling $=($ Summation of $y$ in replicated sampling $) T(j)$

END FOR

END FOR

FOR i FROM 1 TO the number of systematic sampling

Summation of empirical variance in each number of systematic sampling is assigned to be zero

FOR $j$ FROM 1 TO the number of replication

Empirical variance in each replication $=(\text { Estimated } Q \text { in each replication-True value })^{\wedge} 2$;

Summation of empirical variance in each replication

END FOR

Mean of empirical variance=Summation of empirical variance in each replication/The number of replication

The coefficient of error for the empirical true variance=The root of mean of empirical variance/True value END FOR

\subsubsection{Estimations for Parameter $q$ and Variance Approximation of $\hat{Q}$}

As implied by p. 319 into [9], it is obvious that the changing of $M F$ affects the variance extension term given in Eq. (14). In this sense, the covariogram model in Eq. (11) can be adopted for the some measurement functions. The simulation results in Tables $5-16$ show that the covariogram model can be used for the measurement functions given in Eqs. (7), (8) and (10). Note that the integral of function in Eq. (8) is computed by means of the numerical integration in MATLAB 2013a, but the integrals of functions in Eq. (7), (9) and (10) are computed by means of the 'int' function which is a function for the exact calculation of integral in MATLAB 2013a. The covariogram model in Eq. (11) is proposed intuitively by $[28,29]$. We want to use it to check the performance of variance estimation in Eq. (19). By using the covariogram model in Eq. (11), the estimation of $q$ can be said as an open problem for the measurement functions in Eqs. (7)- (10) except to Eq. (7) with $q=0.4$. It is observed from the 
simulation results that the performance of $q$ formula depends on the covariogram model in Eq. (11) and the variance extension term in Eq. (19). For irregular $M F$, such as Eqs. (9)-(10), the estimations of $q$ are unstable, namely, they change according to the sample size. It should be noted that for each sample size, the information on equidistant systematically sampling version of $f$ changes. When we have regular patterns, such as Eqs. (7)-(8), the estimations of $q$ are stable according to the sample size.

Suppose that we have a flexible balloon filled by gas. This three-dimensional balloon can have different shapes after modification done by hands. Thus, each measurement function can be in a class for the functions with parameter $q$. As it is seen, Eqs. (9) and (10) do not have the parameter $q$, but we want to estimate it to get the values of variance estimation precisely for them. The true parameter values of them are accepted to be $q=0.95, q=0.9$, respectively, because it seems that the estimated values of parameter $q$ are around these values under Eqs. (11) and (19).

The estimated values for $q$ of the measurement functions in Eq. (7) with $q=0.8$ and also Eq. (8) with $q=0.4$ and $q=0.8$ would be around the true parameter values. It is seen that the covariogram model in Eq. (11) can not be a very good representative for these $q$ values of two measurement functions. However, when it is considered on the performance of variance estimation, the values of CEMC and CEET for the systematic sampling of Eqs. (7), (8), and (10) contain the same numbers of zero digits at the fraction part at most times under Eqs. (11) and (19). It should be noted that even though MF in Eq. (10) does not have the parameter $q$, the values of CEMC and CEET contain the same number of zero in fraction part when the sample sizes are from 6 to 20 . When $n=5$, there is not enough information due to the irregular form of $M F$. It is seen from the simulation results that the values of CEMC for MF in Eq. (10) are more approximate than that of Eqs. (7), (8) generally, which shows an evidence that we can propose a function without a parameter $q$ which is a neighborhood of functions with parameter $q$. In other words, we have a family for the covariogram functions. This family can be modelled by the covariogram model. This is one of the reasons why the proposed $M F$ must be used. The efficiency for the systematic sampling of $M F$ in Eq. (9) is not as good as that of Eq. (10) because of Eqs. (11) and (19). The covariogram function of Eq. (9) can not be modelled by the covariogram model. The precision of systematic sampling of MF in Eq. (9) should be improved. This shows us that the proposed measurement functions are also used while making a research in the applied science when Eq. (19) is used. As a result, for Eqs. (7), (8) and (10), Eqs. (11) and (19) give the values around CEET, however for Eq. (9) the values of CEMC and CEET do not contain the same numbers of zero digits at the fraction part even if the sample size increases. The fluctuation of CEET is an expected result, because the idea of estimation for parameter $Q$ via the systematic sampling in Eq. (1) is used.

\subsubsection{Confidence Interval of $\hat{Q}$ : Empirical True and Approximation for $\operatorname{Var}(\hat{Q})$}

The theoretical coverage probability of the confidence interval for a parameter $Q$ was $100 \%$. In this sense, the coverage probabilities of confidence intervals based on the coefficient of error for the Matheron's covariogram model are $100 \%$; but the coverage probabilities of confidence intervals based on the coefficient of error for the empirical true variance can not be $100 \%$. It is observed that the coverage probabilities of confidence intervals based on the coefficient of error for the empirical true variance are $95 \%$ approximately. The benefit of using covariogram model with parameter $q$ is that the bandwidth of confidence can be more accurate for a class of measurement functions, such as Eqs. (7), (8) and (10).

\subsubsection{Confidence Interval of $\hat{Q}: \lambda(q, N)$}

CEMC can be approximate to CEET for Eqs. (7), (8) and (10), and so the coverage probabilities of the confidence intervals based on CEMC and CEET, which are called as CPCEMC and CPCEET respectively, can be considered to have similar values, which shows that our investigation on the performance of $\lambda(q, N)$ values is reasonable. In other words, when we look at the performance of confidence interval whether or not it includes the true value, we should focus on CPCEMC and 
CPCEET must give similar results together. In this point, the trustiness of confidence interval is acceptable, because CPCEET is at least $95 \%$ approximately. In all performed simulation, $N$ in $\lambda(q, N)$ is taken to be 2, which shows us that the confidence interval keeps itself on optimal sense. This optimality is supported by CPCEMC and CPCEET, because they have similar results. In other words, suppose that if $N$ is taken to be 3 , the bandwidths of confidence interval in $\lambda(q, 3)$ case are larger than that of $\lambda(q, 2)$. In such a case, we would have a non useful information for the confidence band of $\hat{Q}$. The another point we should focus on is the values in Tables 1 and 2, because the exact values of constant $\lambda(q, N)$ provide useful information for the confidence band. For MF in Eq. (9), since CPCEET is $100 \%$, it seems the constant $\lambda(q, N)$ produces the confidence intervals, which includes the true value of area. However, the accurateness of the constant $\lambda(q, N)$ with the empirical true variance and the approximate variance estimations should be examined together. For the same values of $\lambda(q, N)$, the bandwidths of confidence intervals with CEET are smaller than that of CEMC, which shows us that the bandwidths of confidence intervals constructed with CEET are more accurate than that of CEMC. When we look at Table 16, CPCEMC and CPCEET have same values except that the number of sampling $n$ is equal to 5 . The more number of sampling means the more information gained from the $M F$. This is one of the reasons why the proposed $M F$ must be used. For $M F$ in Eq. (10), it is important to take a value for $q$ such that we can not only estimate the variance of systematic sampling more precisely but also choose a right value for the constant $\lambda(q, N)$. Finally, proposing a class of measurement functions for the researches helps us to control the trustworthiness of the researches in the applied science if the experts in area can propose them correctly.

As a final comment for the simulation section, the covariogram model in Eq. (11) and the variance extension term in Eq. (19) can be used. However, the proposed measurement functions, such as Eqs. (7) - (10), should be systematically sampled so that one can get the more precise decision on the application for the biomedical imaging. As an another solution, Eq. (19) may be reformulated in the framework of fractional Fourier transformation proposed by $[7,34]$.

\subsection{Real Data}

Five different sheep brains which were 12-18 months old were removed from their skull via craniotomy in the laboratory for anatomy. These brains were immersed in formalin (5\%) for 10 days. Brains were scanned with standard T2-weighted 0.5 tesla Magnetic Resonance Imaging (MRI) in the coronal plane with $5 \mathrm{~mm}$ slice thickness. The true values of volume for each brain were obtained by using Archimedean principle repeated in 6 times. The arithematic mean of 6 results for each brain was used as a true value of volume of a brain. After MRI scanned the sheep brains, the area of each digital images for slices of brains was computed by pixel counting. While doing the pixel counting, the edges of each digital images were detected by experts in the anatomy area. After that, they were estimated by the slices in coronal plane. The results are given in Tables 3-4. Tables 3-4 show the true values for the volume $(Q)$, the estimated volume $(\hat{Q})$, the estimated smoothness constant $(\hat{q})$, the estimated coefficient of error $(\hat{c})$ ), the lower bound of the estimated volume $\left(\hat{Q}_{\text {lower }}\right)$,the upper bound of the estimated volume $\left(\hat{Q}_{\text {upper }}\right)$ and the number of slices (Num. Slc.). The area values of each slice obtained from the coronal axis are depicted at the Figure 3. 


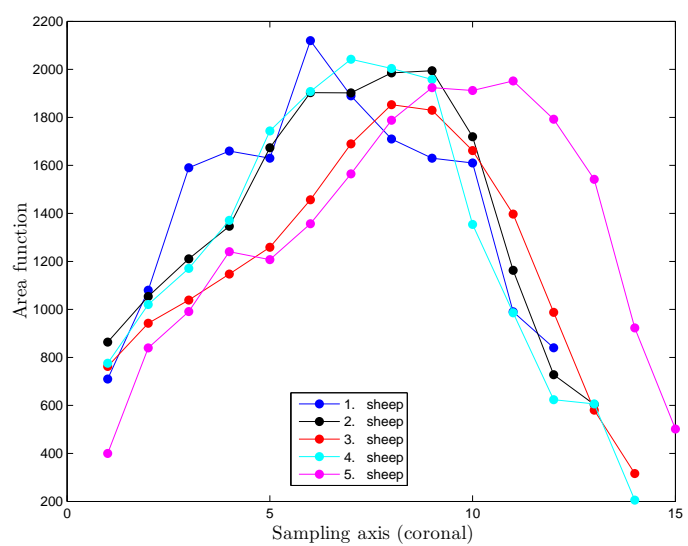

Figure 3. Area functions for each brain.

Table 3. 5 Sheep brain volumes from automatic pixel counting $\left(\mathrm{mm}^{2}\right)$ after determining border of slices with $5 \mathrm{~mm}$ thickness and their confidence intervals for $N=2$ : arithmetic mean of $\hat{q}$ values with $k=2,3,4,5,6,7$ in Eq. (22)

\begin{tabular}{cccccccc}
\hline Brains & $\mathrm{Q}$ & $\hat{Q}$ & $\hat{q}$ & $\hat{c} e$ & $\hat{Q}_{\text {lower }}$ & $\hat{Q}_{\text {upper }}$ & Num. Slc. \\
\hline \hline 1 & 85000 & 87300 & .20 & .0234 & 81289.05 & 93310.95 & 12 \\
2 & 94000 & 90738 & .34 & .0106 & 87731.40 & 93744.60 & 13 \\
3 & 83000 & 84608 & .50 & .0066 & 82722.64 & 86493.36 & 14 \\
4 & 88000 & 88846 & .42 & .0087 & 86318.13 & 91372.87 & 14 \\
5 & 100000 & 99675 & .67 & .0039 & 98351.66 & 100998.34 & 15 \\
\hline
\end{tabular}

When $N$ in Eq. (29) is replaced with $3, \lambda(0.3,3)=3.82689$ and $\lambda(0.34,3)=3.90405$ are found. The confidence intervals of brain 2 for $q=0.3$ and $q=0.34$ are $(87055.68,94420.32)$ and $(86981.44$, 94494.56), respectively. These confidence intervals include the true value of volume for the brain 2 .

Table 4. 5 Sheep brain volumes from automatic pixel counting $\left(\mathrm{mm}^{2}\right)$ after determining border of slices with $5 \mathrm{~mm}$ thickness and their confidence intervals for $N=2: \hat{q}$ values with $k=2$ in Eq. (22)

\begin{tabular}{cccccccc}
\hline Brains & $\mathrm{Q}$ & $\hat{Q}$ & $\hat{q}$ & $\hat{c} e$ & $\hat{Q}_{\text {lower }}$ & $\hat{Q}_{\text {upper }}$ & Num. Slc. \\
\hline \hline 1 & 85000 & 87300 & -.06 & .0234 & 82646.98 & 91953.02 & 12 \\
2 & 94000 & 90738 & .18 & .0131 & 87249.31 & 94226.69 & 13 \\
3 & 83000 & 84608 & .41 & .0075 & 82464.84 & 86751.16 & 14 \\
4 & 88000 & 88846 & .28 & .0105 & 85934.10 & 91756.90 & 14 \\
5 & 100000 & 99675 & .63 & .0041 & 98283.46 & 101066.54 & 15 \\
\hline
\end{tabular}

In table 4 , when the estimated $\hat{q}$ with $k=2$ is taken, the confidence interval includes the true values of volume for each non-vivo brains. It is seen that estimating accurately the parameter $q$ affects the variance estimation and the confidence interval as well. For this reason, $\lambda(q, N)$ values for $N=2,3,4$ in Table 1 are computed. The simulation results show that $N$ should be 2 . The more precise measurement of the slice area is needed for the real data case, because measuring the area of slices more precisely produces the shape of $M F$ more accurately. Having more accurate shape of $M F$ means that the estimated values of parameter $q$ can be determined more accurately. However, it should be noted that the estimation of parameter $q$ except to Eq. (7) with $q=0.4$ is an open problem as well even if the exact form of $M F$ is known. Taking the values of $k$ in $q$ formula and $N$ in the constant $\lambda(q, N)$ are bigger than 2 is not useful due to loss on the information, however we do not have a solution for the real data case. In real data case, we have to come across trying the different values of $k$ and $N$ 
and also the proposed $M F$ must be used to increase the trustworthiness of researches in the applied science. As a result, using two propositions is required.

\section{Conclusions and Discussions}

The studies [9-12,16,19-21,25-27,30] focused on the variance estimation for the systematic sampling. As implied by $[11,15,16]$, the estimation of $q$ is important to avoid the biasedness of the variance estimator in systematic sampling on $\mathbb{R}$. Unbiasedness of variance extension term estimator leads to have the accurate lower and upper bounds of confidence interval for the systematic sampling on $\mathbb{R}$. In fact, the true variance in Eq. (4) is the combination of three components, so we always have biased variance estimation, however for the practical purpose, we are only interested in the variance extension term. It is observed from the simulation results that CEMC and CEET have the values which contain the same numbers of zero digits at the fraction part for the measurement functions in Eqs. (7), (8), (10) at most times under Eqs. (11) and (19). However, the same performance of the formula in Eq. (19) is not observed for the variance approximation of systematic sampling of $M F$ in Eq. (9) even if the number of sample size increases. So, the formulae in Eqs. (11) and Eq. (19) are not appropriate to use. It should be noted that due to the definition of systematic sampling given in Eq. (1), a fluctuation can be observed, so evaluating the performance of CEMC and CEET according to the behaviour of the sample size can not be sensible. However, when the sample size increases, it is logical to expect the information coming from $M F$ will be increased. In this context, Table $5-16$ show that when the sample size increases, the values of CEMC and CEET are decreased. As implied by p. 319 into [9], if a measurement function has irregularities, such as Eqs. (8), (9), and (10), the values of CEMC are high when the sample size is small, because the information is not enough to catch the approximate shape of the three-dimensional object. For the measurement functions in Eqs. (8), (9), and (10), Table 10-16 support this implication into p. 319 of [9]. The CEET values of MF in Eq. (8) with $q=0.4$ can approve the fluctuation when the sample sizes are $5-20$. For other measurement functions, the fluctuation on CEET is not observed, in fact there is a fluctuation, however the effect of sample size covers the fluctuation. Otherwise, proposing a Fourier transformation to model the estimated values of parameter $Q$ would not give the results for CEMC which are approximate to CEET.

A three-dimensional object is represented by a flexible balloon filled by gas. The shape of this balloon can be modified by means of hands. The volume of balloon can change after each modification, however it is not important whether or not there is a changing of the volume of balloon. The modified versions of the balloon are represented by the measurement functions given in Eqs. (7) - (10). The measurement functions in Eqs. (9)-(10) do not have the parameter $q$, however they can be a neighborhood of MF having parameter $q$. Since we have different form of the balloon, we will have a lot of forms of the measurement functions representing the three-dimensional object exactly. In real data case, knowing the exact form of three-dimensional object is impossible. The covariogram functions coming from the $M F$ without $q$ will be neighborhood of the covariogram functions coming from $M F$ with $q$. This is why the covariogram model has the parameter $q$. The true $M F$ with $q$ can not be known exactly due to fact that we can have $M F$ without $q$ which is a neighborhood of $M F$ with $q$. In the other words, we can have approximately the same functions which are different mathematical expressions.

A method showing how to calculate constant $\lambda(q, N)$ is proposed. It is expected that this method can be used as a new tool in Mathematics when it is needed. A package program written in MATLAB $2013 a$ gives the lower and upper levels of confidence and the quantitative values of stereology when the data obtained from a single replication is typed. The program can be supplied on a request. The estimation of $q$ is an open problem even if we know the exact form of the measurement functions except to Eq. (7) with $q=0.4$. In other perspective of our discussion, the covariogram model can not be a very good approximation for the covariogram functions of measurement functions. However, 
Table 5. $\left(1-x^{2}\right)^{q}: q=0.4$

\begin{tabular}{ccccc}
\cline { 2 - 4 }$n$ & $\hat{q}$ & $\widehat{\operatorname{Var}}(\hat{q})$ & $\widehat{M S E}(\hat{q})$ \\
\cline { 2 - 5 } $\mathbf{4 3 5}$ & 0.469211 & 0.000755 & 0.005545 \\
6 & 0.449412 & 0.001009 & 0.003450 \\
7 & 0.438921 & 0.000994 & 0.002509 \\
8 & 0.430876 & 0.000992 & 0.001945 \\
9 & 0.426702 & 0.001029 & 0.001742 \\
10 & 0.422139 & 0.001089 & 0.001580 \\
11 & 0.419202 & 0.001029 & 0.001398 \\
12 & 0.417282 & 0.001049 & 0.001348 \\
13 & 0.414414 & 0.001213 & 0.001421 \\
14 & 0.413815 & 0.001090 & 0.001281 \\
15 & 0.411180 & 0.001210 & 0.001335 \\
16 & 0.411575 & 0.001060 & 0.001194 \\
17 & 0.409010 & 0.001232 & 0.001314 \\
18 & 0.409631 & 0.001119 & 0.001211 \\
19 & 0.409595 & 0.001086 & 0.001178 \\
20 & 0.408593 & 0.001079 & 0.001153 \\
\hline
\end{tabular}
(10). be used. given here and in future.

\section{Appendix A}

Table 6. $\left(1-x^{2}\right)^{q}: q=0.4$

the values of $C E M C$ and $C E E T$ contain the same numbers of zero digits at the fraction part at most times for the measurement functions in Eqs. (7), (8), (10) under Eqs. (11) and (19).

The values of $C P C E M C$ and CPCEET are other criteria to approve the performance of exact values of the constant $\lambda(q, N)$, as observed from the simulation results. A numerical computation for the constant $\lambda(q, N)$ of confidence interval was done by [17]. The more precise values of the constant $\lambda(q, N)$ mean the more precise confidence interval. It is obvious that the proposition of exact calculation has to be preferred, because the computation of the constant $\lambda(q, N)$ proposed by [17] is not as good as the results displayed by Tables 1 and 2, as seen by the conformity between CPCEMC and CPCEET for exact values of $\lambda(q, N)$, especially for the measurement functions in Eqs. (7), (8) and

It is observed that the synthetic data can approve the real data for non-vivo brains if the real data has an exactly similar form with the synthetic data. Since the real data can not be represented by the exactly similar measurement functions, we should prefer to use different $N$ values. For this reason, we chose the different $k$ values for estimation of $q$ in real data. Eq. (22) can produce the negative estimated $\hat{q}$ value for the area function of real data given in Figure 3. The constant $\lambda(q, N)$ values for different $N$ values must be given, because the real data shows that we can need constant $\lambda(q, N)$ values with different $N$. In this context, the edge of digital images has to be found more precisely. Especially, when the edges of images are more irregular, the precision of getting area values for each image has to be decreased significantly. To do this, new edge detection methods proposed by [33] can

The proposed measurement functions should be systematically sampled while conducting a research on the biomedical imaging to increase the information in the decision rule for now. To be able to increase the performance of variance approximation, the fractional Fourier transformation may be applied to get a new variance estimation of the equidistant systematically sampled on $\mathbb{R}$. Other types of $M F$ may also be used. In this case, we need the computational integral techniques. The studies done by $[35,40]$ will be light for the computational integration of other $M F$ types that we will use. For construction of $M F$ via the digital images which have coronal, axial or sagittal directions, the new edge detection methods proposed by [33] may be applied to get more precise area values of slices. We will prepare a free statistical software $R$ package with a macro of Mathematica for all methodologies

\begin{tabular}{ccccc}
\hline$n$ & CEMC & CEET & CPCEMC\% & CPCEET\% \\
\hline 5 & 0.031387 & 0.027150 & 100.0 & 98.8 \\
6 & 0.024935 & 0.021804 & 100.0 & 99.1 \\
7 & 0.020275 & 0.017246 & 100.0 & 99.1 \\
8 & 0.016988 & 0.014385 & 100.0 & 99.3 \\
9 & 0.014433 & 0.011948 & 100.0 & 98.7 \\
10 & 0.012535 & 0.010502 & 100.0 & 99.1 \\
11 & 0.011000 & 0.009178 & 100.0 & 99.5 \\
12 & 0.009751 & 0.008085 & 100.0 & 99.0 \\
13 & 0.008768 & 0.007458 & 100.0 & 99.0 \\
14 & 0.007891 & 0.006564 & 100.0 & 99.1 \\
15 & 0.007211 & 0.006121 & 100.0 & 99.3 \\
16 & 0.006562 & 0.005408 & 100.0 & 99.3 \\
17 & 0.006069 & 0.005181 & 100.0 & 99.2 \\
18 & 0.005580 & 0.004672 & 100.0 & 99.5 \\
19 & 0.005167 & 0.004271 & 100.0 & 99.4 \\
20 & 0.004820 & 0.003961 & 100.0 & 99.3 \\
\hline
\end{tabular}


Table 7. $\left(1-x^{2}\right)^{q}: q=0.8$

\begin{tabular}{cccc}
\hline$n$ & $\hat{q}$ & $\widehat{\operatorname{Var}}(\hat{q})$ & $\widehat{\operatorname{MSE}}(\hat{q})$ \\
\hline 5 & 0.798607 & 0.014187 & 0.014189 \\
6 & 0.821274 & 0.015347 & 0.015800 \\
7 & 0.827312 & 0.016704 & 0.017450 \\
8 & 0.838035 & 0.016471 & 0.017918 \\
9 & 0.835330 & 0.017350 & 0.018599 \\
10 & 0.840301 & 0.017530 & 0.019154 \\
11 & 0.840649 & 0.017641 & 0.019294 \\
12 & 0.839555 & 0.018128 & 0.019693 \\
13 & 0.842700 & 0.017997 & 0.019821 \\
14 & 0.837016 & 0.018080 & 0.019450 \\
15 & 0.838411 & 0.018319 & 0.019794 \\
16 & 0.840756 & 0.018341 & 0.020002 \\
17 & 0.837451 & 0.018479 & 0.019881 \\
18 & 0.831963 & 0.019301 & 0.020323 \\
19 & 0.839645 & 0.019642 & 0.021214 \\
20 & 0.835757 & 0.019321 & 0.020599 \\
\hline
\end{tabular}

Table 9. $\left((1-\cos (x))\left(1-x^{2}\right)\right)^{q}: q=0.4$

\begin{tabular}{cccc}
\hline$n$ & $\hat{q}$ & $\widehat{\operatorname{Var}}(\hat{q})$ & $\widehat{\operatorname{MSE}(\hat{q})}$ \\
\hline 5 & -0.059737 & 0.009231 & 0.220590 \\
6 & 0.257864 & 0.001882 & 0.022085 \\
7 & 0.412975 & 0.000590 & 0.000758 \\
8 & 0.483350 & 0.000779 & 0.007726 \\
9 & 0.501458 & 0.000922 & 0.011216 \\
10 & 0.507921 & 0.001314 & 0.012961 \\
11 & 0.507945 & 0.000837 & 0.012489 \\
12 & 0.507897 & 0.001435 & 0.013076 \\
13 & 0.503777 & 0.000699 & 0.011469 \\
14 & 0.501835 & 0.001445 & 0.011816 \\
15 & 0.497033 & 0.000737 & 0.010153 \\
16 & 0.494786 & 0.001527 & 0.010511 \\
17 & 0.489366 & 0.000710 & 0.008696 \\
18 & 0.488828 & 0.001432 & 0.009323 \\
19 & 0.483590 & 0.000714 & 0.007702 \\
20 & 0.481851 & 0.001531 & 0.008230 \\
\hline
\end{tabular}

Table 8. $\left(1-x^{2}\right)^{q}: q=0.8$

\begin{tabular}{ccccc}
\hline$n$ & CEMC & CEET & CPCEMC\% & CPCEET\% \\
\hline 5 & 0.018256 & 0.022835 & 100.0 & 100.0 \\
6 & 0.012532 & 0.016332 & 100.0 & 100.0 \\
7 & 0.009405 & 0.012425 & 100.0 & 100.0 \\
8 & 0.007190 & 0.009670 & 100.0 & 100.0 \\
9 & 0.005913 & 0.007842 & 100.0 & 100.0 \\
10 & 0.004853 & 0.006570 & 100.0 & 100.0 \\
11 & 0.004053 & 0.005437 & 100.0 & 100.0 \\
12 & 0.003491 & 0.004684 & 100.0 & 100.0 \\
13 & 0.003021 & 0.004037 & 100.0 & 100.0 \\
14 & 0.002674 & 0.003554 & 100.0 & 100.0 \\
15 & 0.002380 & 0.003191 & 100.0 & 100.0 \\
16 & 0.002116 & 0.002814 & 100.0 & 100.0 \\
17 & 0.001885 & 0.002528 & 100.0 & 100.0 \\
18 & 0.001702 & 0.002280 & 100.0 & 100.0 \\
19 & 0.001549 & 0.002043 & 100.0 & 100.0 \\
20 & 0.001429 & 0.001903 & 100.0 & 100.0 \\
\hline
\end{tabular}

Table 10. $\left((1-\cos (x))\left(1-x^{2}\right)\right)^{q}: q=0.4$

\begin{tabular}{ccccc}
\hline$n$ & CEMC & CEET & CPCEMC\% & CPCEET\% \\
\hline 5 & 0.136986 & 0.035193 & 100.0 & 94.6 \\
6 & 0.075842 & 0.056090 & 100.0 & 97.4 \\
7 & 0.048192 & 0.022847 & 100.0 & 98.0 \\
8 & 0.035892 & 0.036584 & 100.0 & 99.1 \\
9 & 0.029172 & 0.017402 & 100.0 & 98.3 \\
10 & 0.024801 & 0.026910 & 100.0 & 99.5 \\
11 & 0.021386 & 0.013797 & 100.0 & 98.6 \\
12 & 0.018852 & 0.020350 & 100.0 & 99.4 \\
13 & 0.016763 & 0.010922 & 100.0 & 98.9 \\
14 & 0.015121 & 0.016248 & 100.0 & 99.7 \\
15 & 0.013697 & 0.009184 & 100.0 & 98.5 \\
16 & 0.012545 & 0.013334 & 100.0 & 99.4 \\
17 & 0.011526 & 0.007842 & 100.0 & 98.9 \\
18 & 0.010623 & 0.011027 & 100.0 & 99.7 \\
19 & 0.009865 & 0.006687 & 100.0 & 98.5 \\
20 & 0.009211 & 0.009652 & 100.0 & 99.6 \\
\hline
\end{tabular}


Table 11. $\left((1-\cos (x))\left(1-x^{2}\right)\right)^{q}: q=0.8$

\begin{tabular}{cccc}
\hline$n$ & $\hat{q}$ & $\widehat{\operatorname{Var}}(\hat{q})$ & $\widehat{M S E}(\hat{q})$ \\
\hline 5 & -0.127902 & 0.025653 & 0.886655 \\
6 & 0.259307 & 0.009587 & 0.301935 \\
7 & 0.471353 & 0.008843 & 0.116851 \\
8 & 0.591335 & 0.008365 & 0.051906 \\
9 & 0.667866 & 0.009158 & 0.026618 \\
10 & 0.712836 & 0.010045 & 0.017643 \\
11 & 0.743469 & 0.010628 & 0.013824 \\
12 & 0.769775 & 0.011272 & 0.012186 \\
13 & 0.782195 & 0.012278 & 0.012595 \\
14 & 0.794971 & 0.013111 & 0.013137 \\
15 & 0.802343 & 0.013406 & 0.013412 \\
16 & 0.810423 & 0.013491 & 0.013599 \\
17 & 0.814130 & 0.013994 & 0.014194 \\
18 & 0.822037 & 0.014498 & 0.014984 \\
19 & 0.822426 & 0.014351 & 0.014854 \\
20 & 0.822911 & 0.015298 & 0.015823 \\
\hline
\end{tabular}

Table 13. $\exp \left(-\sin \left(-x^{3}\right)\right): q=0.95$

\begin{tabular}{cccc}
\hline$n$ & $\hat{q}$ & $\widehat{\operatorname{Var}}(\hat{q})$ & $\widehat{\operatorname{MSE}(\hat{q})}$ \\
\hline 5 & -0.088096 & 0.050826 & 1.128469 \\
6 & 0.041988 & 0.031897 & 0.856384 \\
7 & 0.189917 & 0.018652 & 0.596379 \\
8 & 0.320166 & 0.008843 & 0.405534 \\
9 & 0.449291 & 0.007235 & 0.257945 \\
10 & 0.549659 & 0.004972 & 0.165245 \\
11 & 0.639576 & 0.002916 & 0.099279 \\
12 & 0.714919 & 0.001908 & 0.057171 \\
13 & 0.776112 & 0.001817 & 0.032055 \\
14 & 0.827798 & 0.001763 & 0.016697 \\
15 & 0.868178 & 0.001724 & 0.008419 \\
16 & 0.899500 & 0.001604 & 0.004154 \\
17 & 0.928089 & 0.001326 & 0.001806 \\
18 & 0.942607 & 0.001128 & 0.001183 \\
19 & 0.959425 & 0.001025 & 0.001114 \\
20 & 0.967014 & 0.000817 & 0.001106 \\
\hline
\end{tabular}

Table 12. $\left((1-\cos (x))\left(1-x^{2}\right)\right)^{q}: q=0.8$

\begin{tabular}{ccccc}
\hline$n$ & CEMC & CEET & CPCEMC\% & CPCEET\% \\
\hline 5 & 0.179574 & 0.070757 & 100.0 & 95.8 \\
6 & 0.098853 & 0.058929 & 100.0 & 98.7 \\
7 & 0.056666 & 0.040635 & 100.0 & 100.0 \\
8 & 0.037906 & 0.035454 & 100.0 & 100.0 \\
9 & 0.027210 & 0.025762 & 100.0 & 100.0 \\
10 & 0.021142 & 0.023491 & 100.0 & 100.0 \\
11 & 0.016973 & 0.018652 & 100.0 & 100.0 \\
12 & 0.014014 & 0.016925 & 100.0 & 100.0 \\
13 & 0.011669 & 0.013700 & 100.0 & 100.0 \\
14 & 0.010083 & 0.012682 & 100.0 & 100.0 \\
15 & 0.008732 & 0.010624 & 100.0 & 100.0 \\
16 & 0.007799 & 0.010070 & 100.0 & 100.0 \\
17 & 0.006862 & 0.008591 & 100.0 & 100.0 \\
18 & 0.006093 & 0.008067 & 100.0 & 100.0 \\
19 & 0.005530 & 0.007070 & 100.0 & 100.0 \\
20 & 0.005030 & 0.006753 & 100.0 & 100.0 \\
\hline
\end{tabular}

Table 14. $\exp \left(-\sin \left(-x^{3}\right)\right): q=0.95$

\begin{tabular}{ccccc}
\hline$n$ & CEMC & CEET & CPCEMC\% & CPCEET\% \\
\hline 5 & 0.123899 & 0.042779 & 100.0 & 100.0 \\
6 & 0.096852 & 0.020019 & 100.0 & 100.0 \\
7 & 0.068757 & 0.008892 & 100.0 & 100.0 \\
8 & 0.046235 & 0.003329 & 100.0 & 100.0 \\
9 & 0.031395 & 0.001138 & 100.0 & 100.0 \\
10 & 0.022537 & 0.000614 & 100.0 & 100.0 \\
11 & 0.016419 & 0.000538 & 100.0 & 100.0 \\
12 & 0.012289 & 0.000462 & 100.0 & 100.0 \\
13 & 0.009477 & 0.000366 & 100.0 & 100.0 \\
14 & 0.007494 & 0.000282 & 100.0 & 100.0 \\
15 & 0.006081 & 0.000220 & 100.0 & 100.0 \\
16 & 0.005047 & 0.000184 & 100.0 & 100.0 \\
17 & 0.004236 & 0.000158 & 100.0 & 100.0 \\
18 & 0.003677 & 0.000132 & 100.0 & 100.0 \\
19 & 0.003202 & 0.000117 & 100.0 & 100.0 \\
20 & 0.002850 & 0.000103 & 100.0 & 100.0 \\
\hline
\end{tabular}


Table 15. $(5 / 112)\left(-54 x^{4}-25 x^{3}+48 x^{2}+\right.$ $25 x+6): q=0.9$

\begin{tabular}{cccc}
\cline { 2 - 4 }$n$ & $\hat{q}$ & $\widehat{\operatorname{Var}}(\hat{q})$ & $\widehat{M S E}(\hat{q})$ \\
\cline { 2 - 5 } 540.119701 & 0.021089 & 0.629956 \\
6 & 0.349198 & 0.018577 & 0.321959 \\
7 & 0.506408 & 0.017147 & 0.172062 \\
8 & 0.607155 & 0.017009 & 0.102768 \\
9 & 0.677396 & 0.017249 & 0.066802 \\
10 & 0.720912 & 0.016863 & 0.048935 \\
11 & 0.765776 & 0.016458 & 0.034474 \\
12 & 0.786878 & 0.015944 & 0.028741 \\
13 & 0.815932 & 0.015711 & 0.022778 \\
14 & 0.838310 & 0.015351 & 0.019157 \\
15 & 0.856894 & 0.015623 & 0.017481 \\
16 & 0.865002 & 0.016006 & 0.017231 \\
17 & 0.883260 & 0.015086 & 0.015367 \\
18 & 0.887106 & 0.015674 & 0.015840 \\
19 & 0.896326 & 0.015430 & 0.015444 \\
20 & 0.904643 & 0.014824 & 0.014845 \\
\hline
\end{tabular}

Table 16. $(5 / 112)\left(-54 x^{4}-25 x^{3}+48 x^{2}+\right.$ $25 x+6): q=0.9$

\begin{tabular}{ccccc}
\hline$n$ & CEMC & CEET & CPCEMC\% & CPCEET\% \\
\hline 5 & 0.131979 & 0.061047 & 100.0 & 97.7 \\
6 & 0.076664 & 0.044234 & 100.0 & 100.0 \\
7 & 0.047065 & 0.031891 & 100.0 & 100.0 \\
8 & 0.032165 & 0.024913 & 100.0 & 100.0 \\
9 & 0.023411 & 0.019979 & 100.0 & 100.0 \\
10 & 0.018146 & 0.015962 & 100.0 & 100.0 \\
11 & 0.014053 & 0.013222 & 100.0 & 100.0 \\
12 & 0.011617 & 0.011093 & 100.0 & 100.0 \\
13 & 0.009464 & 0.009315 & 100.0 & 100.0 \\
14 & 0.007890 & 0.007985 & 100.0 & 100.0 \\
15 & 0.006690 & 0.006992 & 100.0 & 100.0 \\
16 & 0.005872 & 0.006274 & 100.0 & 100.0 \\
17 & 0.005015 & 0.005386 & 100.0 & 100.0 \\
18 & 0.004499 & 0.004952 & 100.0 & 100.0 \\
19 & 0.003983 & 0.004410 & 100.0 & 100.0 \\
20 & 0.003544 & 0.003891 & 100.0 & 100.0 \\
\hline
\end{tabular}

Acknowledgments: I am very grateful to Professor Luis M. Cruz-Orive to sincerely invite me, to Dr. Marta García-Fiñana for sending the codes in $R$, and to Dr. Niyazi Acer for providing kindly the data. I am also so indebted to the Council of Higher Education to give the funding for my research. I am indebted to James $F$. Peters from University of Manitoba, Winnipeg, Canada for critical reading. I would like to thank sincerely to four referees. Without their helps, this paper was not improved. I also wish to thank my nice parent. In the memory of my nice brother...

\section{References}

1. Abramowitz, M.; Stegun, I.A. Handbook of Mathematical Functions, Dover, New York, 1970, 1058p.

2. Acer N.; Şahin B.; Usanmaz M.; Tatoğlu H.; Irmak Z. Comparison of Point Counting and Planimetry Methods for the Assessment of Cerebellar Volume in Human using Magnetic Resonance Imaging: a stereological study. Surg Radiol Anat 2008, 30, 335 - 339.

3. Acer, N.; Şahin, B.; Emirzeoğlu, M.; Uzun, A.; İncesu, L.; Bek, Y.; Bilgiç, S.; Kaplan, S. Stereological Estimation of the Orbital Volume: A Criterion Standard Study. Journal of Craniofacial Surgery, 2009, 20, 921-938.

4. Acer, N.; Çankaya, M.N.; İşçi, Ö.; Baş, O.; Çamurdanoğlu, M.; Turgut, M. Estimation of Cerebral Surface Area using Vertical Sectioning and Magnetic Resonance Imaging: A Stereological Study. Brain Research, 2010, 1310, 29 -36.

5. Akbaş, H.; Şahin, B.; Eroğlu, L.; Odac1, E.; Bilgiç, S.; Kaplan, S.; Uzun, A.; Ergür, H.; Bek, Y. Estimation of Breast Prosthesis Volume by the Cavalieri Principle Using Magnetic Resonance Images. Aesthetic plastic sur. 2004, 28, 275 - 280.

6. Baddeley, A.J.; Vedel Jensen, E.B. Stereology for Statisticians, Monographs on Statistics and Applied Probability, Chapman \& Hall/CRC, USA. 2005, 380p.

7. Baleanu D.; Wu G.C.; Duan J.S. Some Analytical Techniques in Fractional Calculus: Realities and Challenges, Discontinuity and Complexity in Nonlinear Physical Systems, Volume 6 of the series Nonlinear Systems and Complexity. 2013, 35-62.

8. Bellhouse, D.R.; Krishnaiah, P.R.; Rao, C.R. (Editors), Sampling: Handbook of Statistics, Vol. 6, North-Holland, Amsterdam. 1988, 125-145.

9. Cruz-Orive, L.M. On the Precision of Systematic Sampling:A Review of Matheron's Transitive Methods. Journal of Microscopy. 1989, 153, 315-333.

10. Cruz-Orive, L.M. Systematic Sampling in Stereology. Bull. Intern. Statis. Inst., Proceedinngs 49th Session, Firenze. 1993, 55, 451-468.

11. Cruz-Orive, L.M. A General Variance Predictor for Cavalieri Slices. Journal of Microscopy. 2006, 222, 158-165. 
12. Cruz-Orive, L.M. Variance predictors for isotropic geometric sampling, with applications in forestry. Statistical Methods E Applications. 2012, 22, 3-31.

13. Çankaya, M.N. Stereological Estimation and Inference with Applications. Unpublished Master Thesis, Council of Higher Education. 2010, 168p.

14. Eriksen N.; Rostrup E.; Andersen K.; Lauritzen M.J.; Larsen V.A.; Dreier J.P.; Strong A.J.; Hartings J.A.; Fabricius M.; Pakkenberg B. Application of stereological estimates in patients with severe head injuries using CT and MR scanning images. British Institute of Radiology. 2010, 83, 307-317.

15. García-Fiñana, M.; Cruz-Orive, L.M.; Mackay Clare E.; Pakkenberg B.; Roberts, N. Comparison of MR Imaging Against Physical Sectioning to Estimate the Volume of Human Cerebral Compartments. NeuroImage. 2003, 18, 505-516.

16. García-Fiñana, M.; Cruz-Orive, L.M. Improved Variance Prediction for Systematic Sampling on R. Taylor and Francis Group, Statistics. 2004, 38, 243-272.

17. García-Fiñana, M. Confidence intervals in Cavalieri Sampling. Journal of Microscopy. 2006, 222, $146-157$.

18. García-Fiñana, M.; Keller, S.S.; Roberts, N. Confidence Intervals for the Volume of Brain Structures in Cavalieri Sampling with Local Errors. Journal of Neuroscience Methods. 2009, 179, 71-77.

19. Gual Arnau, X.; Cruz-Orive, L.M. Variance prediction under systematic sampling with geometric probes. Adv. Appl. Prob. 1998, 30, 889-903.

20. Gundersen, H.J.G.; Jensen, E.B. The Efficiency of Systematic Sampling in Stereology and its Prediction. Journal of Microscopy. 1987, 147, 229-263.

21. Gundersen, H.J.G.; Jensen, E.B.V.; Kiêu, K.; Nielsen, J. The Efficiency of Systematic Sampling in Stereology - Reconsidered. Journal of Microscopy. 1999, 193, 199-211.

22. Hall, P.; Ziegel J. Distributions estimators and confidence intervals for stereological volumes, Biometrika. 2011, 98, 417-431.

23. Howard, M.A.; Roberts N.; García-Fiñana, M.; Cowell, P.E. Volume Estimation of Prefrontal Cortical Subfields using MRI and Stereology. Brain Research Protocols. 2003, 10, 125-138.

24. Hussain, Z.; Roberts, N.; Whitehouse, G.H.; García-Fiñana, M.; Percy D. Estimation of Breast Volume and its Variation During the Menstrual Cycle using MRI and Stereology. The British Journal of Radioology. 1999, 72, 236-245.

25. Kellerer, A.M. Exact formulae for the precision of systematic sampling. J. Microsc. 1989, 153, $285-300$.

26. Kiêu, K. Three Lectures on Systematic Geometric Sampling., Memoirs 13. Department of Theoretical Statistics, University of Aarush. 1997, 100p.

27. Kiêu K.; Souchet, S.; Istas, J. Precision of Systematic Sampling and Transitive Methods, Journal of Statistical Planning and Inference. 1999, 77, 263-279.

28. Matheron, G. Variables régionalisées et leur estimation [Les], Masson et CIE, éditeurs. 1965.

29. Matheron, G. The Theory of Regionalized Variables and Its Applications. Les Cahires du Centre de Morphologie Mathématique de Fontainebleau, No. 5. Ecole Nationale Supérieure des Mines de Paris. 1971, F. Fontainebleau.

30. Mattfeldt, T. The accuracy of one-dimensional systematic sampling. J. Microsc. 1989, 153, 301-313.

31. Maudsley, R.; García-Fiñana, M. Sampling Intensity with Fixed Precision When Estimating Volume of Human Brain Compartments. Image Anal Stereol. 2008, 27, 143-149.

32. McNulty V.; Cruz-Orive L.M.; Roberts N.; Holmes C.J.; Gual-Arnau, X. Estimation of Brain Compartment Volume from MR Cavalieri Slices. Journal of Computer Assisted Tomography. 2000, 24, 466-477.

33. Peters J.F. Computational Proximity Excursions in the Topology of Digital Images, Intelligent Systems Reference Library Vol. 102, Springer, Switzerland. 2016, 433p.

34. Poularikas A.D. Transforms and Applications Handbook, Third Edition. CRC Press: Taylor and Fracis Group. 2010.

35. Qin Y.M.; Shi Y.G. A new approximate method to conjugacies between a family of unimodal interval maps. J. Comput. Complex. Appl. 2016, 2, 163-169.

36. Roberts, N.; Cruz-Orive, L.M.; Reid, M.K.; Brodie, D.A.; Bourne, M.; Edwards, H.T. Unbiased Estimation of Human Body Composition by the Cavalieri Method Using Magnetic Resonance Imaging, Journal of Microscopy. 1993, 171, 239-253.

37. Roberts N.; Pubdephat M.J.; McNulty V. The Benefit of Stereology for Quantitative Radiology, The British Journal of Radiology. 2000, 73, 679-697. 
526

527

528

529

530

531

532

533

38. Şahin, B.; Emirzeoğlu, M.; Uzun, A.; İncesu, L.; Bek, Y.; Bilgiç, S.; Kaplan, S. Unbiased Estimation of the Liver Volume by the Cavalieri Principle using Magnetic Resonance Images. European Journal of Radiology. 2003, 47, 164-170.

39. Şahin, B.; Ergür, H. Assessment of the Optimum Section Thickness for the Estimation of Liver Volume Using Magnetic Resonance Images: A Stereological Gold Standard Study. European Journal of Radiology. 2005, 57, 96-101.

40. Zeng Y. Approximate solutions of three integral equations by the new Adomian decomposition method. J. Comput. Complex. Appl. 2016, 2, 38-43.

(c) 2016 by the author; licensee Preprints, Basel, Switzerland. This article is an open access article distributed under the terms and conditions of the Creative Commons Attribution (CC-BY) license (http:/ / creativecommons.org/licenses/by/4.0/). 